\title{
AHRR methylation in heavy smokers: associations with smoking, lung cancer risk, and lung cancer mortality
}

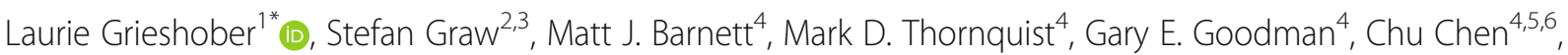
Devin C. Koestler ${ }^{2 \dagger}$, Carmen J. Marsit ${ }^{3+}$ and Jennifer A. Doherty ${ }^{1,4+}$

\begin{abstract}
Background: A low level of methylation at cg05575921 in the aryl-hydrocarbon receptor repressor (AHRR) gene is robustly associated with smoking, and some studies have observed associations between cg05575921 methylation and increased lung cancer risk and mortality. To prospectively examine whether decreased methylation at cg05575921 may identify high risk subpopulations for lung cancer screening among heavy smokers, and mortality in cases, we evaluated associations between cg05575921 methylation and lung cancer risk and mortality, by histotype, in heavy smokers.

Methods: The $\beta$-Carotene and Retinol Efficacy Trial (CARET) included enrollees ages 45-69 with $\geq 20$ pack-year smoking histories and/or occupational asbestos exposure. A subset of CARET participants had cg05575921 methylation available from HumanMethylationEPIC assays of blood collected on average 4.3 years prior to lung cancer diagnosis in cases. Cg05575921 methylation $\beta$-values were treated continuously for a 10\% methylation decrease and as quintiles, where quintile 1 (Q1, referent) represents high methylation and Q5, low methylation. We used conditional logistic regression models to examine lung cancer risk overall and by histotype in a nested casecontrol study including 316 lung cancer cases (diagnosed through 2005) and 316 lung cancer-free controls matched on age ( \pm 5 years), sex, race/ethnicity, enrollment year, current/former smoking, asbestos exposure, and follow-up time. Mortality analyses included 372 lung cancer cases diagnosed between 1985 and 2013 with available methylation data. We used Cox proportional hazards models to examine mortality overall and by histotype.
\end{abstract}

Results: Decreased cg05575921 methylation was strongly associated with smoking, even in our population of heavy smokers. We did not observe associations between decreased pre-diagnosis cg05575921 methylation and increased lung cancer risk, overall or by histotype. We observed linear increasing trends for lung cancer-specific mortality across decreasing cg05575921 methylation quintiles for adenocarcinoma and small cell carcinoma ( $P$ trends $=0.01$ and 0.04 , respectively).

(Continued on next page)

\footnotetext{
* Correspondence: laurie.grieshober@hci.utah.edu

${ }^{\dagger}$ Devin C. Koestler, Carmen J. Marsit and Jennifer A. Doherty contributed equally to this work.

'Department of Population Health Sciences, Huntsman Cancer Institute, University of Utah, 2000 Circle of Hope Drive, Room 4746, Salt Lake City, UT 84112, USA

Full list of author information is available at the end of the article
}

(c) The Author(s). 2020 Open Access This article is licensed under a Creative Commons Attribution 4.0 International License, which permits use, sharing, adaptation, distribution and reproduction in any medium or format, as long as you give appropriate credit to the original author(s) and the source, provide a link to the Creative Commons licence, and indicate if changes were made. The images or other third party material in this article are included in the article's Creative Commons licence, unless indicated otherwise in a credit line to the material. If material is not included in the article's Creative Commons licence and your intended use is not permitted by statutory regulation or exceeds the permitted use, you will need to obtain permission directly from the copyright holder. To view a copy of this licence, visit http://creativecommons.org/licenses/by/4.0/ The Creative Commons Public Domain Dedication waiver (http://creativecommons.org/publicdomain/zero/1.0/) applies to the data made available in this article, unless otherwise stated in a credit line to the data. 
(Continued from previous page)

Conclusions: In our study of heavy smokers, decreased cg05575921 methylation was strongly associated with smoking but not increased lung cancer risk. The observed association between cg05575921 methylation and increased mortality in adenocarcinoma and small cell histotypes requires further examination. Our results do not support using decreased cg05575921 methylation as a biomarker for lung cancer screening risk stratification.

Keywords: Lung cancer, Epidemiology, Biomarkers/serum biomarkers, Methylation, AHRR, CARET, Mortality

\section{Background}

Exposure to cigarette smoke is associated with altered DNA methylation at thousands of individual cytosineguanine dinucleotide (CpG) sites across the genome in both blood and lung tissue based on results from at least 73 epigenome-wide association studies (EWAS) [1]. The most consistent association for any CpG with smoking is decreased methylation at cg05575921 in the aryl hydrocarbon receptor repressor gene $(A H R R)$, which has been associated with cigarette smoking in whole blood samples in at least 30 EWAS [1]. The cg05575921 locus typically shows the largest absolute difference in methylation by cigarette smoking relative to other individual CpGs [2-11]. Longitudinal studies have shown that decreased methylation of cg05575921 persists in former smokers compared to never smokers, and that methylation gradually increases with time since cessation $[5,11,12]$.

Cg05575921 is located in an AHRR gene enhancer, and decreased methylation in this region results in increased AHRR gene expression in both blood [13, 14] and lung tissue [15-17]. Greater AHRR expression inhibits the aryl-hydrocarbon receptor, which among other functions, regulates toxicity of polycyclic aromatic hydrocarbons (PAHs) [18]. Since cigarette smoke contains PAHs, it has been hypothesized that decreased AHRR methylation induced by cigarette smoking may be a mediator in lung cancer development [19]. Several epidemiologic studies support this hypothesis and report that a low level of cg05575921 methylation is associated with increased lung cancer risk [4, 9, 19-22]. However, these reports all include light and never smokers. While decreased cg05575921 methylation has been reported to be associated with all-cause mortality $[9,12]$, the relationship between pre-diagnosis cg05575921 methylation and mortality in lung cancer cases is less clear. One casecohort study reported increased lung cancer-specific mortality [23], but results were not presented by histotype, which could limit the examination of associations among tumor subgroups with known differences in treatment response and mortality. To our knowledge, no studies to date have examined associations with prediagnosis cg05575921 methylation and mortality, allcause or lung cancer-specific, among lung cancer cases.

Since a low level of cg05575921 methylation is highly correlated with increased smoking exposure, and has been reported to be associated with lung cancer risk, it is an appealing marker to examine for risk stratification for lung cancer screening. Since 2014, the United States Preventive Services Task Force (USPSTF) has recommended annual lung cancer screening for individuals aged 55-80 years who have at least 30 pack-year smoking histories and are current or former smokers who quit within the past 15 years [24]. An updated $2020 \mathrm{draft}$ USPSTF recommendation statement broadens screening eligibility to include those aged $50-80$ with 20 or more pack-year smoking histories, still among current or former smokers who quit within the past 15 years [25]. In order for a biomarker to improve lung cancer screening risk stratification by minimizing false-positive screens, it must be associated with lung cancer risk among individuals who are eligible for screening. We sought to disentangle the relationships between cg05575921 methylation, lung cancer risk, and lung cancer mortality in a nested case-control study of heavy smokers generally representative of a lung cancer screening-eligible population.

\section{Methods}

Our study includes a subset of participants from the multicenter $\beta$-Carotene and Retinol Efficacy Trial (CARET) [26]. CARET was a randomized, doubleblinded, placebo-controlled trial designed to assess the safety and efficacy of daily $\beta$-carotene and retinyl palmitate supplementation in heavy smokers at high risk of developing lung cancer [26-28]. From 1985 to 1994, CARET enrolled 14,254 men and women ages 50-69 years who were current or former smokers (quit $\leq 6$ years prior to enrollment) with $\geq 20$ pack-year cigarette smoking histories. Men with occupational asbestos exposure ages 45-69 years who were current or former smokers (quit $\leq 15$ years prior to enrollment) were also enrolled $(n=4060)$. Smoking status, smoking history, and other risk factors were collected via annual questionnaires. Whole blood samples were collected at visits between 1994 and 1997. The intervention was stopped in 1996 due to higher lung cancer incidence and overall mortality rates in the intervention versus placebo arm.

Within our larger matched case-control study designed to examine genetic factors and lung cancer risk described in [29], we generated whole-genome DNA 
methylation data for 350 lung cancer cases identified during active follow-up between 1985 and 2005, and one matched control per case. The case-control pairs were matched on enrollment characteristics including age $( \pm 4$ years) and smoking status, as well as sex, race/ethnicity, enrollment year ( \pm 2 years), and history of occupational asbestos exposure. Controls were cancer-free at least as long as their corresponding case through 2005.

DNA was extracted from whole blood using QIAGEN QIAmp DNA Blood Midi Kits $(n=348$ cases, $n=347$ controls) and 5PRIME ArchivePure DNA Purification Kits ( $n=2$ cases, $n=3$ controls). DNA methylation was assayed in a single batch using the Illumina HumanMethylationEPIC BeadArray at the University of Southern California Epigenomics Core Facility following standardized protocols from Illumina, Inc. We performed data quality control, preprocessing, and Noob+ $\beta$-mixture quantile normalization using the minfi and wateRmelon Bioconductor packages [30,31], described in detail previously [32]. Analytical $\beta$-values, representing percent methylation, were obtained for the cg05575921 locus.

Since blood was collected at post-enrollment study visits, and DNA methylation is influenced by age and smoking status, we re-matched among the 350 casecontrol pairs using age ( \pm 5 years) and smoking status (current or former) at blood draw, rather than at enrollment, as well as sex, race/ethnicity, enrollment year $( \pm 2$ years), asbestos exposure, and duration of follow-up. A total of 322 case-control pairs were able to be rematched, but three pairs missing data on body mass index (BMI) were removed, resulting in 319 pairs in our previous study [32]. For the present analysis, we included the three pairs missing BMI, but we discovered that there were six mismatched pairs that were removed for the present analysis. Analyses examining cg05575921 methylation and risk of lung cancer therefore include 316 matched case-control pairs, with blood collected on average 4.3 years prior to diagnosis for the cases. Mortality analyses were performed for all 350 lung cancer cases diagnosed through 2005, plus 22 controls who developed lung cancer during passive follow-up from 2005 to 2013; blood was collected on average 4.9 years prior to diagnosis for this larger case group.

\section{Statistical analysis}

We categorized cg05575921 percent methylation into quintiles, with quintile 1 (Q1, referent) containing the top $20 \%$ of percent methylation values (i.e., hypermethylation), and Q5 containing the lowest 20\% of percent methylation values (i.e., hypomethylation). Cut points for cg05575921 quintile methylation for the lung cancer risk analyses are based on the distribution of cg05575921 methylation in the controls. We used ordinal linear regression to assess linear trends of association between cg05575921 methylation quintiles and continuous participant characteristics including age, BMI, cigarettes per day in current smokers, pack years smoked, and years since cessation in former smokers. We assessed linear trends in proportions of strata for discrete participant characteristics, including race, sex, smoking status, and occupational asbestos exposure, as well as stage and histotype (adenocarcinoma, squamous cell carcinoma, or small cell carcinoma) across cg05575921 methylation quintiles using CochranArmitage Trend tests, or Fisher's Exact tests for variables with at least $50 \%$ of cells containing expected counts of less than five per cell.

We evaluated associations between continuous decreasing cg05575921 methylation and lung cancer risk using multivariable-adjusted logistic regression models conditioned on matching factors. In addition to a priori selected adjustment for continuous age at blood draw (to reduce residual confounding by age) and methylation-derived estimated blood cell type proportions [33, 34], adjustment variables were assessed for inclusion based on biologic plausibility and/or if their addition to age- and estimated cell type-adjusted conditional logistic regression models for all lung cancer cases resulted in $a \geq 10 \%$ change in the estimated odds ratio for either quintile or continuous 10\% decreased cg05575921 methylation. Final risk models were adjusted for age at blood draw, estimated blood cell proportions, and cigarettes per day at blood draw. We performed the same analysis restricted to the 242 matched pairs where both the case and control would have been eligible for lung cancer screening based on age (55-80 years) and smoking ( $\geq 30$ pack years; current or quit $<15$ years) per the 2014 USPSTF recommendation statement.

For mortality analyses, quintile cg05575921 percent methylation cut points were based on the distribution including all 372 lung cancer cases. We evaluated associations between decreasing pre-diagnosis cg05575921 methylation and lung cancer-specific and all-cause mortality using multivariable-adjusted Cox proportional hazards models with follow-up defined as time between lung cancer diagnosis and death or December 31, 2013, whichever occurred first. We included a strata variable for early, late, or unknown stage to allow for differing baseline hazards since stage at diagnosis is strongly associated with mortality [35]. Continuous age, sex, methylation-derived estimated blood cell type proportions [33, 34], and time between blood draw and diagnosis were a priori selected for adjustment, and additional variables were included based on biologic plausibility and/or if their addition to a priori variable-adjusted Cox proportional hazards models for all lung cancer cases resulted in $\mathrm{a} \geq 10 \%$ change in the estimated hazard ratio 
(all-cause or lung cancer-specific) for either quintile or continuous $10 \%$ decreased cg05575921 methylation. Final mortality models were adjusted for age at blood draw, sex, estimated blood cell proportions, time between blood draw and diagnosis, smoking status, and years since smoking cessation at blood draw.

We performed a sensitivity analysis excluding the three pairs where either the case or control had DNA extracted by the 5PRIME method. We also examined the possibility of interaction by sex in the mortality models, overall and by histotype, to ensure sound adjustment for sex as a confounder and not an effect modifier in our models. All analyses were performed in SAS 9.4 (Cary, NC). Statistical tests were two-sided and statistical significance testing was performed at a nominal level of $P<0.05$.

\section{Results}

We observed highly statistically significant linear trends of increasing proportions of current smokers across decreasing cg05575921 methylation quintiles in both lung cancer cases and controls $\left(P_{\text {case }}=2 \times 10^{-22}, P_{\text {control }}=4 \times\right.$ $10^{-25}$; Table 1). Striking differences in the proportions of current smokers were observed in quintile five (Q5) compared to Q1 in both cases (90\% vs $24 \%$ ) and controls (89\% vs $22 \%)$. Similar trends were observed across increasing quintiles with greater total years smoked $\left(P_{\text {case }}=0.03, P_{\text {control }}=1 \times 10^{-8}\right)$, fewer years since cessation in former smokers $\left(P_{\text {case }}=0.002, P_{\text {control }}=0.001\right)$, and more cigarettes smoked per day in current smokers $\left(P_{\text {case }}=8 \times 10^{-5}, P_{\text {control }}=0.04\right)$. We observed linear associations with increasing quintiles for increasing pack years (only statistically significant among controls: $P_{\text {con- }}$ trol $\left.=0.004 ; P_{\text {case }}=0.15\right)$, decreasing BMI $\left(P_{\text {case }}=0.004\right.$, $P_{\text {control }}=0.002$ ), and age at blood draw (only statistically significant among cases: $P_{\text {case }}=7 \times 10^{-5} ; P_{\text {control }}=0.07$ ). We observed decreasing proportions of individuals with asbestos exposure across increasing quintiles $\left(P_{\text {case }}=\right.$ $\left.0.05 ; P_{\text {control }}=0.003\right)$. We observed similar linear trends across decreasing cg05575921 methylation quintiles in the full 372 cases examined in the mortality analyses (Additional file 1: Table S1).

Although strong and highly statistically significant associations were observed between decreased cg05575921 methylation and aspects of smoking exposure (Table 1; Additional file 1: Tables S1-S2), there were no clear associations between decreased cg05575921 methylation and lung cancer risk overall or by histotype in the 316 matched case-control pairs after controlling for age, estimated cell type, and cigarettes per day at blood draw (Table 2). Neither odds ratios nor linear trends reached statistical significance. While there was a nonstatistically significant greater than two-fold increased risk of adenocarcinoma in Q2 and Q5 compared to Q1, there was no linear association $(P=0.50)$. All odds ratios for squamous cell carcinoma were below one, but they were statistically imprecise. Similar patterns were observed in the 242 case-control pairs where both members of the case-control pair would have been eligible for lung cancer screening per the 2014 USPSTF recommendations, with the exception of small cell histotype in which a borderline linear association emerged $(P$-trend $=$ 0.05 ; Table 3$)$. The screening-eligible small cell histotype quintile estimates became unstable due to small counts, but in the continuous model each $10 \%$ decrease in cg05575921 methylation was associated with a reduced small cell lung cancer risk (Odds Ratio $(\mathrm{OR})=$ 0.51, 95\% CI: $0.28-0.93)$. We did not observe interactions by sex.

In mortality analyses, decreasing cg05575921 methylation was borderline-statistically significantly associated with increased lung cancer-specific and all-cause mortality for all histotypes combined ( $P$-trends $=0.05$ and 0.06 , respectively; Table 4). These associations were driven by the associations in adenocarcinoma and small cell histotypes; no association was observed for squamous cell carcinoma. Among adenocarcinoma cases, we observed linear associations between decreasing cg05575921 methylation quintiles and increased lung cancer-specific mortality $(P=0.01$; Q5 vs Q1 HR $=2.32$, 95\% CI: 1.12 4.82) and all-cause mortality ( $P=0.01$; Q 5 vs Q $1 \mathrm{HR}=$ 2.37, 95\% CI: 1.20-4.71). Each continuous 10\% decrease in cg05575921 methylation was associated with a $21 \%$ greater risk of death in adenocarcinoma cases (lung cancer-specific 95\%CI: 1.03-1.43; all-cause 95\% CI: 1.03-1.41). Among small cell cases, we observed a linear association between decreasing cg05575921 methylation quintiles and increased lung cancer-specific mortality $(P=0.04$; $\mathrm{Q} 5$ vs Q1 HR $=3.68,95 \% \mathrm{CI}: 1.32-10.25)$, and although the all-cause mortality quintile results were generally similar, the linear trend was not statistically significant $(P=0.09)$. We did not observe evidence for statistical interaction by sex in any of our mortality models.

Associations excluding individuals with 5PRIME extracted DNA were similar to the main risk and mortality results including them, respectively (Additional file 1: Tables S3-S5).

\section{Discussion}

To our knowledge, our study is the first to examine associations between pre-diagnosis AHRR cg05575921 methylation and lung cancer risk and mortality by histotype among smokers at high risk of lung cancer. We observed that cg05575921 methylation differed dramatically by smoking exposure even among this population of heavy smokers, with mean pack years of 59.3 in cases and 54.2 in controls. Though strong and highly 
Table 1 Characteristics of lung cancer cases and controls by quintiles of cg05575921 percent methylation

\begin{tabular}{|c|c|c|c|c|c|c|c|}
\hline \multirow[b]{2}{*}{ Quintile range } & All & \multicolumn{2}{|c|}{ (hyper-methylated) } & Q3 & Q4 & \multicolumn{2}{|l|}{$\begin{array}{l}\text { Q5 } \\
\text { (hypo-methylated) }\end{array}$} \\
\hline & $34-98$ & 84-98 & $73-84$ & $64-73$ & $56-64$ & $34-56$ & \\
\hline \multicolumn{8}{|l|}{ Lung cancer cases $^{a}$} \\
\hline & $(n=316)$ & $(n=59)$ & $(n=78)$ & $(n=59)$ & $(n=49)$ & $(n=71)$ & $P$-trend ${ }^{\mathrm{b}}$ \\
\hline cg05575921, \% methylation; mean (SD) & $\begin{array}{l}69.1 \\
(14.6)\end{array}$ & $89.6(3.8)$ & $78.0(3.1)$ & $68.2(2.7)$ & $60.0(2.4)$ & $49.2(5.0)$ & - \\
\hline Age at blood draw, years; mean (SD) & $64.5(5.5)$ & $65.9(5.8)$ & $65.5(5.5)$ & $64.9(5.7)$ & $63.8(4.9)$ & $62.5(5.3)$ & $7 \times 10^{-5}$ \\
\hline Current smoker; No. (\%) & $205(65)$ & $14(24)$ & $38(49)$ & $44(75)$ & $45(92)$ & $64(90)$ & $2 \times 10^{-22}$ \\
\hline Years since smoking cessation ${ }^{c}$; mean (SD) & $6.6(4.8)$ & $8.1(5.2)$ & $6.4(4.6)$ & $4.3(3.9)$ & $5.0(2.2)$ & $3.7(2.6)$ & 0.002 \\
\hline Pack years; mean (SD) & $\begin{array}{l}59.3 \\
(22.5)\end{array}$ & $55.3(18.4)$ & $\begin{array}{l}59.0 \\
(25.1)\end{array}$ & $\begin{array}{l}60.2 \\
(22.3)\end{array}$ & $\begin{array}{l}60.7 \\
(23.8)\end{array}$ & $61.3(22.2)$ & 0.15 \\
\hline Average cigarettes per day ${ }^{\mathrm{d}}$; mean (SD) & $\begin{array}{l}23.3 \\
(12.4)\end{array}$ & $17.6(11.6)$ & $\begin{array}{l}17.1 \\
(11.6)\end{array}$ & $\begin{array}{l}23.7 \\
(12.1)\end{array}$ & $\begin{array}{l}25.4 \\
(13.1)\end{array}$ & $26.4(11.4)$ & $8 \times 10^{-5}$ \\
\hline Total years smoked; mean (SD) & $44.3(6.7)$ & $42.1(8.0)$ & $44.2(6.9)$ & $45.5(6.2)$ & $45.3(6.0)$ & $44.7(5.8)$ & 0.03 \\
\hline $\mathbf{B M I}^{\mathrm{e}}, \mathrm{kg} / \mathrm{m}^{2} ;$ mean $(\mathrm{SD})$ & $27.6(4.9)$ & $29.2(4.8)$ & $27.6(4.7)$ & $27.6(4.7)$ & $27.7(5.8)$ & $26.3(4.4)$ & 0.004 \\
\hline Race, white; No. (\%) & $307(97)$ & $58(98)$ & $73(94)$ & $59(100)$ & $47(96)$ & $70(99)$ & $0.56^{f}$ \\
\hline Sex, female; No. (\%) & $109(34)$ & $21(36)$ & $25(32)$ & $26(44)$ & $15(31)$ & $22(31)$ & 0.60 \\
\hline Intervention arm, assigned to active; No. (\%) & $164(52)$ & $30(51)$ & $41(53)$ & $31(53)$ & $29(59)$ & $33(46)$ & 0.79 \\
\hline Asbestos exposure; No. (\%) & $51(16)$ & $15(25)$ & $14(18)$ & $7(12)$ & $5(10)$ & $10(14)$ & 0.05 \\
\hline Stage; No. (\%) & & & & & & & $0.66^{9}$ \\
\hline Early stage (I/II) & $72(23)$ & $13(18)$ & $21(29)$ & $8(11)$ & $10(14)$ & $20(28)$ & \\
\hline Late stage (III/IV) & $195(62)$ & $38(19)$ & $44(23)$ & $42(22)$ & $31(16)$ & $40(21)$ & \\
\hline Unknown stage & $49(16)$ & $8(16)$ & $13(27)$ & $9(18)$ & $8(16)$ & $11(22)$ & \\
\hline \multicolumn{8}{|l|}{ Histotype; No. (\%) } \\
\hline Adenocarcinoma & $132(42)$ & $28(47)$ & $35(45)$ & $20(34)$ & $18(37)$ & $31(44)$ & \\
\hline Squamous cell carcinoma & $103(33)$ & $15(25)$ & $23(29)$ & $16(27)$ & $20(41)$ & $29(41)$ & \\
\hline Small cell carcinoma & $71(22)$ & $15(25)$ & $19(24)$ & $19(32)$ & $9(18)$ & $9(13)$ & \\
\hline Any death (through 2015); No. (\%) & $304(96)$ & $58(98)$ & $73(94)$ & $56(95)$ & $49(100)$ & $68(96)$ & $0.92^{f}$ \\
\hline $\begin{array}{l}\text { Years between blood draw and diagnosis; } \\
\text { mean (SD) }\end{array}$ & $4.3(2.5)$ & $3.6(2.5)$ & $4.3(2.5)$ & $4.5(2.4)$ & $4.4(2.6)$ & $4.8(2.3)$ & 0.02 \\
\hline \multicolumn{8}{|l|}{ Controls } \\
\hline & $(n=316)$ & $(n=63)$ & $(n=64)$ & $(n=63)$ & $(n=63)$ & $(n=63)$ & $P$-trend ${ }^{b}$ \\
\hline cg05575921, \% methylation; mean (SD) & $\begin{array}{l}69.3 \\
(14.8)\end{array}$ & $90.8(4.0)$ & $78.3(2.9)$ & $67.6(2.6)$ & $60.0(2.0)$ & $49.4(5.0)$ & - \\
\hline Age at blood draw, years; mean (SD) & $63.5(5.7)$ & $64.6(6.0)$ & $63.6(5.2)$ & $64.2(5.8)$ & $61.7(5.9)$ & $63.4(5.4)$ & 0.07 \\
\hline Current smoker; No. (\%) & $205(65)$ & $14(22)$ & $28(44)$ & $49(78)$ & $58(92)$ & $56(89)$ & $4 \times 10^{-25}$ \\
\hline Years since smoking cessation ${ }^{c}$; mean (SD) & $6.6(6.4)$ & $9.0(7.9)$ & $5.6(4.3)$ & $4.1(3.4)$ & $2.8(2.6)$ & $3.9(4.5)$ & 0.001 \\
\hline Pack years; mean (SD) & $\begin{array}{l}54.2 \\
(24.1)\end{array}$ & $48.4(23.5)$ & $\begin{array}{l}53.6 \\
(18.8)\end{array}$ & $\begin{array}{l}52.9 \\
(23.2)\end{array}$ & $\begin{array}{l}53.7 \\
(25.8)\end{array}$ & $62.2(27.2)$ & 0.004 \\
\hline Average cigarettes per day ${ }^{\mathrm{d}}$; mean (SD) & $\begin{array}{l}21.5 \\
(10.7)\end{array}$ & $20.9(8.6)$ & $\begin{array}{l}19.1 \\
(10.9)\end{array}$ & $21.2(9.7)$ & $19.7(9.9)$ & $25.0(11.9)$ & 0.04 \\
\hline Total years smoked; mean (SD) & $42.5(7.4)$ & $38.6(8.0)$ & $41.0(6.9)$ & $43.8(6.9)$ & $43.2(7.3)$ & $45.8(6.0)$ & $1 \times 10^{-8}$ \\
\hline $\mathbf{B M I}^{\mathbf{e}}, \mathrm{kg} / \mathrm{m}^{2} ;$ mean $(\mathrm{SD})$ & $28.1(5.5)$ & $29.2(5.0)$ & $29.3(6.9)$ & $27.6(5.4)$ & $27.7(5.4)$ & $26.6(4.3)$ & 0.002 \\
\hline Race, white; No. (\%) & $307(97)$ & $62(98)$ & $59(92)$ & $61(97)$ & $62(98)$ & $63(100)$ & $0.19^{f}$ \\
\hline Sex, female; No. (\%) & 109 (34) & $27(43)$ & $20(31)$ & $21(33)$ & $24(38)$ & $17(27)$ & 0.19 \\
\hline Intervention arm, assigned to active; No. (\%) & $168(53)$ & $27(43)$ & $32(50)$ & $36(57)$ & $38(60)$ & $35(56)$ & 0.07 \\
\hline Asbestos exposure; No. (\%) & $51(16)$ & $18(29)$ & $12(19)$ & $5(8)$ & $12(19)$ & $4(6)$ & 0.003 \\
\hline
\end{tabular}


Table 1 Characteristics of lung cancer cases and controls by quintiles of cg05575921 percent methylation (Continued)

\begin{tabular}{|c|c|c|c|c|c|c|c|}
\hline \multirow[b]{2}{*}{ Quintile range } & All & \multicolumn{4}{|c|}{ (hyper-methylated) } & \multicolumn{2}{|c|}{ (hypo-methylated) } \\
\hline & $34-98$ & 84-98 & $73-84$ & $64-73$ & $56-64$ & $34-56$ & \\
\hline Any death (through 2015); №. (\%) & $187(59)$ & $33(52)$ & $33(52)$ & $40(63)$ & $41(65)$ & $40(63)$ & 0.07 \\
\hline \multicolumn{8}{|c|}{$\begin{array}{l}\text { Abbreviations: BMI body mass index, NSCLC non-small cell lung cancer, NSCLC, NOS non-small cell lung } \\
\text { a"Lung cancer cases" includes adenocarcinoma, squamous cell, and small cell, as well as } 10 \text { cases for wh } \\
\text { or missing } \\
\text { b Linear trend tested using ordinal linear regression for continuous variables and Cochran-Armitage Trer } \\
\text { cg05575921 methylation quintiles } \\
\text { cReported for individuals reporting former smoking status at blood draw ( } n=111 \text { case-control pairs) } \\
\text { 'Reported for individuals reporting current smoking status at blood draw ( } n=205 \text { case-control pairs) } \\
\text { eBMl is missing for } 1 \text { case and } 2 \text { controls } \\
\text { fFisher's Exact test used due to at least } 50 \% \text { of cells containing expected counts of less than } 5 \text { per cell } \\
\text { 'Linear trend by Cochran-Armitage Trend test for known stage only (early versus late; } n=293 \text { cases) }\end{array}$} \\
\hline
\end{tabular}

statistically significant associations were observed for lower cg05575921 methylation and greater smoking exposure in our study and in others [2-11], we did not observe that lower cg05575921 methylation was associated with an increased risk of lung cancer risk overall or by histotype. However, we observed that among lung cancer cases, decreased pre-diagnosis cg055759921 methylation was associated with increased mortality for adenocarcinoma and small cell, but not squamous cell lung cancer.

In prior epidemiologic publications, low levels of cg05575921 methylation have been associated with increased risks of lung cancer [4, 9, 19-22]. These reports include never and light smokers, and results have not been presented by histotype. In the population-based study by Bojesen et al. of approximately $23 \%$ never smokers and current/former smokers with mean smoking histories of fewer than 40 pack years, an over fourfold increased risk of lung cancer for individuals in the lowest versus highest methylation quintiles (95\% CI: 2.31-10.30) was observed after adjusting for smoking status, cigarettes per day, and pack years [9]. In four publications reporting on combinations of study populations from up to five nested case-control studies, with each individual nested case-control study comprised of 63 to 367 pairs, statistically significant $40-60 \%$ increased risks of lung cancer per standard deviation decrease in cg05575921 methylation were reported [4, 19, 21, 22]. These results maintained statistical significance after adjustment for smoking for all but one study, which reported a statistically significant $63 \%$ increased risk that was attenuated and no longer statistically significant after controlling for smoking features (e.g., smoking status, pack years, comprehensive smoking index) [22]. In this study, cases had 20 mean pack years while controls averaged nine [22]. Our models of lung cancer risk in heavy smokers per standard deviation decrease in

Table 2 Lung cancer risk ${ }^{a}$ by cg05575921 percent methylation for all lung cancer cases and by histotype

\begin{tabular}{|c|c|c|c|c|c|c|c|c|c|c|c|c|}
\hline \multirow[b]{2}{*}{$\begin{array}{l}\text { cg05575921 } \\
\text { methylation \% }\end{array}$} & \multicolumn{3}{|c|}{ All lung cancer cases ${ }^{b}$} & \multicolumn{3}{|c|}{ Adenocarcinoma } & \multicolumn{3}{|c|}{ Squamous cell carcinoma } & \multicolumn{3}{|c|}{ Small cell } \\
\hline & $\begin{array}{l}\text { Control } \\
\mathrm{n}\end{array}$ & $\begin{array}{l}\text { Case } \\
\mathrm{n}\end{array}$ & $\begin{array}{l}\text { OR }(95 \% \\
\mathrm{Cl})\end{array}$ & $\begin{array}{l}\text { Control } \\
\mathrm{n}\end{array}$ & $\begin{array}{l}\text { Case } \\
\mathrm{n}\end{array}$ & $\begin{array}{l}\text { OR }(95 \% \\
\mathrm{Cl})\end{array}$ & $\begin{array}{l}\text { Control } \\
\mathbf{n}\end{array}$ & $\begin{array}{l}\text { Case } \\
\mathbf{n}\end{array}$ & $\begin{array}{l}\text { OR }(95 \% \\
\mathrm{Cl})\end{array}$ & $\begin{array}{l}\text { Control } \\
\mathrm{n}\end{array}$ & $\begin{array}{l}\text { Case } \\
\mathrm{n}\end{array}$ & OR $(95 \% \mathrm{Cl})$ \\
\hline $\begin{array}{l}\text { Continuous } 10 \% \\
\text { decrease }\end{array}$ & 316 & 316 & $\begin{array}{l}0.93(0.79 \\
1.10)\end{array}$ & 132 & 132 & $\begin{array}{l}1.10(0.85 \\
1.42)\end{array}$ & 103 & 103 & $\begin{array}{l}0.77(0.54 \\
1.11)\end{array}$ & 71 & 71 & $\begin{array}{l}0.70(0.46 \\
1.07)\end{array}$ \\
\hline $\begin{array}{l}\text { Q1 (highest; hyper- } \\
\text { methylated) }\end{array}$ & 63 & 59 & Ref & 33 & 28 & Ref & 11 & 15 & Ref & 17 & 15 & Ref \\
\hline Q2 & 64 & 78 & $\begin{array}{l}1.52(0.81 \\
2.83)\end{array}$ & 23 & 35 & $\begin{array}{l}2.41(0.90 \\
6.46)\end{array}$ & 26 & 23 & $\begin{array}{l}0.32(0.07 \\
1.54)\end{array}$ & 13 & 19 & $\begin{array}{l}2.40(0.54 \\
10.60)\end{array}$ \\
\hline Q3 & 63 & 59 & $\begin{array}{l}0.95(0.47 \\
1.93)\end{array}$ & 27 & 20 & $\begin{array}{l}1.48(0.47, \\
4.63)\end{array}$ & 17 & 16 & $\begin{array}{l}0.17(0.03 \\
1.11)\end{array}$ & 16 & 19 & $\begin{array}{l}1.08(0.25 \\
4.60)\end{array}$ \\
\hline Q4 & 63 & 49 & $\begin{array}{l}0.60(0.28 \\
1.27)\end{array}$ & 32 & 18 & $\begin{array}{l}0.95(0.31 \\
2.89)\end{array}$ & 22 & 20 & $\begin{array}{l}0.16(0.02 \\
1.05)\end{array}$ & 8 & 9 & $\begin{array}{l}0.30(0.04 \\
2.42)\end{array}$ \\
\hline \multirow[t]{2}{*}{$\begin{array}{l}\text { Q5 (lowest; hypo- } \\
\text { methylated) }\end{array}$} & 63 & 71 & $\begin{array}{l}1.03(0.49 \\
2.16)\end{array}$ & 17 & 31 & $\begin{array}{l}2.58(0.77, \\
8.61)\end{array}$ & 27 & 29 & $\begin{array}{l}0.23(0.04 \\
1.37)\end{array}$ & 17 & 9 & $\begin{array}{l}0.49(0.07 \\
3.22)\end{array}$ \\
\hline & \multicolumn{2}{|l|}{$P$-trend } & 0.38 & \multicolumn{2}{|l|}{$P$-trend } & 0.50 & \multicolumn{2}{|l|}{$P$-trend } & 0.26 & \multicolumn{2}{|l|}{$P$-trend } & 0.12 \\
\hline
\end{tabular}

Abbreviations: Cl confidence interval, NSCLC non-small cell lung cancer, NSCLC, NOS non-small cell lung cancer, not otherwise specified, OR Odds ratio aLogistic regression model results, conditioned on matching factors (age at blood draw \pm 5 years, smoking status, sex, race, asbestos, enrollment year \pm 2 years, and time at risk) and adjusted for age at blood draw, estimated cell type, and cigarettes per day at blood draw

"All lung cancer cases" includes adenocarcinoma, squamous cell, and small cell, as well as 10 cases for whom histotype was NSCLC, NOS; other NSCLC; unknown or missing 
Table 3 Lung cancer risk $k^{a}$ by cg05575921 percent methylation, restricted to 2014 USPSTF $^{\mathrm{b}}$ lung cancer screening-eligible pairs

\begin{tabular}{|c|c|c|c|c|c|c|c|c|c|c|c|c|}
\hline \multirow[b]{2}{*}{$\begin{array}{l}\text { cg05575921 } \\
\text { methylation \% }\end{array}$} & \multicolumn{3}{|c|}{ All lung cancer cases ${ }^{c}$} & \multicolumn{3}{|c|}{ Adenocarcinoma } & \multicolumn{3}{|c|}{ Squamous cell carcinoma } & \multicolumn{3}{|c|}{ Small cell } \\
\hline & $\begin{array}{l}\text { Control } \\
\mathbf{n}\end{array}$ & $\begin{array}{l}\text { Case } \\
\mathrm{n}\end{array}$ & OR $(95 \% \mathrm{Cl})$ & $\begin{array}{l}\text { Control } \\
\mathrm{n}\end{array}$ & $\begin{array}{l}\text { Case } \\
\mathrm{n}\end{array}$ & OR $(95 \% \mathrm{Cl})$ & $\begin{array}{l}\text { Control } \\
\mathrm{n}\end{array}$ & $\begin{array}{l}\text { Case } \\
\mathrm{n}\end{array}$ & OR $(95 \% \mathrm{Cl})$ & $\begin{array}{l}\text { Control } \\
\mathrm{n}\end{array}$ & $\begin{array}{l}\text { Case } \\
\mathbf{n}\end{array}$ & OR $(95 \% \mathrm{Cl})$ \\
\hline $\begin{array}{l}\text { Continuous } 10 \% \\
\text { decrease }\end{array}$ & 242 & 242 & $\begin{array}{l}0.90 \\
(0.74,1.08)\end{array}$ & 98 & 98 & $\begin{array}{l}1.08 \\
(0.79,1.46)\end{array}$ & 82 & 82 & $\begin{array}{l}0.76 \\
(0.51,1.12)\end{array}$ & 53 & 53 & $\begin{array}{l}0.51 \\
(0.28,0.93)\end{array}$ \\
\hline $\begin{array}{l}\text { Q1 (highest; hyper- } \\
\text { methylated) }\end{array}$ & 40 & 43 & Ref & 21 & 21 & Ref & 7 & 11 & Ref & 11 & 10 & Ref \\
\hline Q2 & 51 & 59 & $\begin{array}{l}1.05 \\
(0.51,2.16)\end{array}$ & 19 & 22 & $\begin{array}{l}1.39 \\
(0.42,4.62)\end{array}$ & 21 & 21 & $\begin{array}{l}0.27 \\
(0.04,1.88)\end{array}$ & 9 & 16 & $\begin{array}{l}2.84 \\
(0.32,25.21)\end{array}$ \\
\hline Q3 & 51 & 44 & $\begin{array}{l}0.77 \\
(0.33,1.79)\end{array}$ & 21 & 16 & $\begin{array}{l}1.46 \\
(0.35,6.16)\end{array}$ & 14 & 12 & $\begin{array}{l}0.12 \\
(0.01,1.42)\end{array}$ & 13 & 12 & $\begin{array}{l}0.48 \\
(0.05,4.46)\end{array}$ \\
\hline Q4 & 46 & 40 & $\begin{array}{l}0.55 \\
(0.23,1.34)\end{array}$ & 22 & 14 & $\begin{array}{l}1.06 \\
(0.28,4.05)\end{array}$ & 17 & 17 & $\begin{array}{l}0.13 \\
(0.01,1.41)\end{array}$ & 6 & 7 & $\begin{array}{l}0.01 \\
(0.00,0.56)\end{array}$ \\
\hline \multirow[t]{2}{*}{$\begin{array}{l}\text { Q5 (lowest; hypo- } \\
\text { methylated) }\end{array}$} & 54 & 56 & $\begin{array}{l}0.77 \\
(0.33,1.78)\end{array}$ & 15 & 25 & $\begin{array}{l}1.87 \\
(0.47,7.46)\end{array}$ & 23 & 21 & $\begin{array}{l}0.17 \\
(0.02,1.50)\end{array}$ & 14 & 8 & $\begin{array}{l}0.52 \\
(0.04,6.22)\end{array}$ \\
\hline & $P$-trend & & 0.29 & $P$-trend & & 0.55 & $P$-trend & & 0.23 & $P$-trend & & 0.05 \\
\hline
\end{tabular}

Abbreviations: Cl confidence interval, NSCLC non-small cell lung cancer, NSCLC, NOS non-small cell lung cancer, not otherwise specified, OR odds ratio, USPSTF United States Preventive Services Task Force

aLogistic regression model results, conditioned on matching factors (age at blood draw \pm 5 years, smoking status, sex, race, asbestos, enrollment year \pm 2 years, and time at risk) and adjusted for age at blood draw, estimated cell type, and cigarettes per day at blood draw

bIndividuals aged 55-80 with at least 30 pack-year smoking histories who are current or former smokers who had quit within the past 15 years

"All lung cancer cases" includes adenocarcinoma, squamous cell, and small cell, as well as 9 cases for whom histotype was NSCLC, NOS; other NSCLC; unknown or missing

cg05575921 methylation were similar to the continuous $10 \%$ decrease model results shown in Table 2, with an $\mathrm{OR}=0.91(95 \%$ CI: $0.71-1.16)$ for the 316 case-control pairs after controlling for matching factors, age, estimated cell type, and cigarettes per day at blood draw.

In a study that performed a supplementary analysis restricting to the 2014 USPSTF screening eligible smokers, a non-statistically significant 1.2 -fold increased risk of lung cancer per standard deviation decrease in cg05575921 methylation was observed after adjustment for age, sex, pack years, and time since quitting [20]. Again, there were large differences in smoking exposure by case control status, with mean pack years of 34 for cases and 13 for controls [20]. These results are in contrast to our results per standard deviation decrease in cg05575921 methylation, which were similar to the continuous $10 \%$ decrease model results shown in Table 3, with $\mathrm{OR}=0.85$ (95\% CI: 0.65-1.13) in the 2422014 USPSTF screening-eligible pairs after controlling for matching factors, age, estimated cell type, and cigarettes per day at blood draw. An update to the 2014 USPSTF screening guidelines is in process, with the $2020 \mathrm{draft}$ USPSTF recommendation statement broadening eligibility by age ( $50-80$ years) and smoking history (at least a 20 pack-year smoking history) [25]. Based on the 2020 draft USPSTF recommendation, 93\% of the case-control pairs in our study would have been eligible for screening, and thus, our findings reflect the expected associations among that group.
Consistent with our observation that decreased prediagnosis cg05575921 methylation was associated with increased mortality in heavy smoker lung cancer cases, a case-cohort study with 60 fatal lung cancer cases in a subcohort of 1565 participants observed a multivariableadjusted 1.56-fold increased hazard of lung cancerspecific death per 5\% lower pre-diagnosis cg05575921 methylation (95\% CI: 1.30-1.87) [23]. Histotype-specific results were not presented.

Decreased blood cg05575921 methylation is time- and dose-dependent on exposure to cigarette smoking, with cg05575921 methylation gradually increasing after a smoker quits smoking $[11,19,36]$. Two studies of former smokers have reported that cg05575921 methylation levels increase to never-smoker levels on average 10-22 years after cessation [19, 36], while two other studies report that decreased cg05575921 methylation persists 30-35 years post-cessation [11, 37]. Differences in length and condition of blood storage [38, 39], DNA extraction method $[38,40]$, and methylation quantification method $[15,41]$ may contribute to differences in cg05575921 methylation distributions across studies. Fortunately, such between-study differences do not tend to affect differential methylation detection across individuals on a per-study basis [15, 38-40]. This is supported by consistent replication of strong associations between low cg0557921 methylation with smoking features across studies [2-11], regardless of storage or processing. 
Table 4 Mortality ${ }^{a}$ by cg05575921 percent methylation for all lung cancer cases and by histotype

\begin{tabular}{|c|c|c|c|c|c|c|c|c|c|c|c|c|}
\hline \multirow[b]{2}{*}{$\begin{array}{l}\text { cg05575921 } \\
\text { methylation \% }\end{array}$} & \multicolumn{3}{|c|}{ All lung cancer cases ${ }^{b}$} & \multicolumn{3}{|c|}{ Adenocarcinoma } & \multicolumn{3}{|c|}{ Squamous cell carcinoma } & \multicolumn{3}{|c|}{ Small cell } \\
\hline & Deaths & Total & $\begin{array}{l}\text { HR }(95 \% \\
\mathrm{Cl})\end{array}$ & Deaths & Total & $\begin{array}{l}\mathrm{HR}(95 \% \\
\mathrm{Cl})\end{array}$ & Deaths & Total & $\begin{array}{l}\mathrm{HR}(95 \% \\
\mathrm{Cl})\end{array}$ & Deaths & Total & HR $(95 \% \mathrm{Cl})$ \\
\hline \multicolumn{13}{|c|}{ Lung cancer-specific mortality } \\
\hline $\begin{array}{l}\text { Continuous } 10 \% \\
\text { decrease }\end{array}$ & 313 & 372 & $\begin{array}{l}1.08(0.98 \\
1.19)\end{array}$ & 117 & 148 & $\begin{array}{l}1.21(1.03 \\
1.43)\end{array}$ & 94 & 115 & $\begin{array}{l}0.98(0.82 \\
1.17)\end{array}$ & 77 & 81 & $\begin{array}{l}1.20(0.93 \\
1.54)\end{array}$ \\
\hline $\begin{array}{l}\text { Q1 (highest; hyper- } \\
\text { methylated) }\end{array}$ & 59 & 74 & Ref & 23 & 31 & Ref & 15 & 19 & Ref & 16 & 19 & Ref \\
\hline Q2 & 61 & 74 & $\begin{array}{l}0.93(0.64 \\
1.36)\end{array}$ & 26 & 35 & $\begin{array}{l}1.11(0.58 \\
2.11)\end{array}$ & 20 & 23 & $\begin{array}{l}1.02(0.48 \\
2.14)\end{array}$ & 12 & 13 & $\begin{array}{l}1.01(0.37 \\
2.70)\end{array}$ \\
\hline Q3 & 63 & 75 & $\begin{array}{l}1.12(0.75 \\
1.67)\end{array}$ & 21 & 28 & $\begin{array}{l}1.09(0.54 \\
2.19)\end{array}$ & 12 & 17 & $\begin{array}{l}0.89(0.39 \\
2.03)\end{array}$ & 24 & 24 & $\begin{array}{l}1.79(0.79 \\
4.02)\end{array}$ \\
\hline Q4 & 65 & 74 & $\begin{array}{l}1.13(0.75 \\
1.71)\end{array}$ & 23 & 27 & $\begin{array}{l}1.97(0.95 \\
4.10)\end{array}$ & 23 & 27 & $\begin{array}{l}0.86(0.39 \\
1.92)\end{array}$ & 14 & 14 & $\begin{array}{l}1.01(0.35 \\
2.88)\end{array}$ \\
\hline \multirow[t]{2}{*}{$\begin{array}{l}\text { Q5 (lowest; hypo- } \\
\text { methylated) }\end{array}$} & 65 & 75 & $\begin{array}{l}1.46(0.95 \\
2.22)\end{array}$ & 24 & 27 & $\begin{array}{l}2.32(1.12 \\
4.82)\end{array}$ & 24 & 29 & $\begin{array}{l}1.07(0.49, \\
2.36)\end{array}$ & 11 & 11 & $\begin{array}{l}3.68(1.32 \\
10.25)\end{array}$ \\
\hline & \multicolumn{2}{|l|}{$P$-trend } & 0.05 & \multicolumn{2}{|l|}{$P$-trend } & 0.01 & \multicolumn{2}{|l|}{$P$-trend } & 1.00 & \multicolumn{2}{|l|}{$P$-trend } & 0.04 \\
\hline \multicolumn{13}{|l|}{ All-cause mortality } \\
\hline $\begin{array}{l}\text { Continuous } 10 \% \\
\text { decrease }\end{array}$ & 357 & 372 & $\begin{array}{l}1.07(0.97 \\
1.17)\end{array}$ & 137 & 148 & $\begin{array}{l}1.21(1.03, \\
1.41)\end{array}$ & 113 & 115 & $\begin{array}{l}0.97(0.82 \\
1.14)\end{array}$ & 80 & 81 & $\begin{array}{l}1.14(0.89 \\
1.47)\end{array}$ \\
\hline $\begin{array}{l}\text { Q1 (highest; hyper- } \\
\text { methylated) }\end{array}$ & 73 & 74 & Ref & 30 & 31 & Ref & 19 & 19 & Ref & 19 & 19 & Ref \\
\hline Q2 & 69 & 74 & $\begin{array}{l}0.87(0.61 \\
1.24)\end{array}$ & 31 & 35 & $\begin{array}{l}1.05(0.58, \\
1.89)\end{array}$ & 23 & 23 & $\begin{array}{l}1.10(0.56 \\
2.20)\end{array}$ & 12 & 13 & $\begin{array}{l}0.82(0.31 \\
2.18)\end{array}$ \\
\hline Q3 & 71 & 75 & $\begin{array}{l}1.07(0.74 \\
1.56)\end{array}$ & 24 & 28 & $\begin{array}{l}1.08(0.56 \\
2.11)\end{array}$ & 17 & 17 & $\begin{array}{l}1.11(0.53 \\
2.31)\end{array}$ & 24 & 24 & $\begin{array}{l}1.45(0.66 \\
3.17)\end{array}$ \\
\hline Q4 & 70 & 74 & $\begin{array}{l}1.01(0.68 \\
1.49)\end{array}$ & 25 & 27 & $\begin{array}{l}1.65(0.83 \\
3.29)\end{array}$ & 26 & 27 & $\begin{array}{l}0.78(0.36 \\
1.66)\end{array}$ & 14 & 14 & $\begin{array}{l}0.83(0.30 \\
2.28)\end{array}$ \\
\hline \multirow[t]{2}{*}{$\begin{array}{l}\text { Q5 (lowest; hypo- } \\
\text { methylated) }\end{array}$} & 74 & 75 & $\begin{array}{l}1.42(0.96 \\
2.10)\end{array}$ & 27 & 27 & $\begin{array}{l}2.37(1.20 \\
4.71)\end{array}$ & 28 & 29 & $\begin{array}{l}1.04(0.50 \\
2.17)\end{array}$ & 11 & 11 & $\begin{array}{l}2.95(1.09 \\
7.96)\end{array}$ \\
\hline & \multicolumn{2}{|l|}{$P$-trend } & 0.06 & \multicolumn{2}{|l|}{$P$-trend } & 0.01 & \multicolumn{2}{|l|}{$P$-trend } & 0.76 & \multicolumn{2}{|l|}{$P$-trend } & 0.09 \\
\hline
\end{tabular}

Abbreviations: Cl confidence Interval, NSCLC non-small cell lung cancer, NSCLC, NOS non-small cell lung cancer, not otherwise specified, HR hazard ratio ${ }^{a}$ Cox proportional hazards model results adjusted for age at blood draw, sex, years between blood draw and lung cancer diagnosis, and years since quit smoking at blood draw. All models include early, late, or unknown stage as a strata variable

b "All lung cancer cases" includes adenocarcinoma, squamous cell carcinoma, and small cell cases as well as not otherwise specified non-small cell lung cancer (NSCLC, NOS; $n=16)$ and unknown/no pathology $(n=12)$

A major strength of our study is that the population was at high risk of lung cancer due to high levels of cigarette smoke exposure. CARET selection was based on pack years smoked and time since cessation, and cases and controls were matched on current versus former smoking status at blood draw. While matching on smoking status may have ultimately limited our ability to see differences in risk and mortality with a marker that is so strongly related to smoking, our goal was to evaluate whether this marker provided information for lung cancer risk stratification above and beyond the effect of smoking.

\section{Conclusions}

Although cg05575921 is a robust marker of cigarette smoking exposure, our results suggest that low levels of cg05575921 methylation are not associated with an increased risk of lung cancer in heavy smokers, and thus do not support using this marker for risk stratification for lung cancer screening among high-risk individuals. Additional research is needed to inform on whether decreased pre-diagnosis cg05575921 methylation is associated with mortality above and beyond smoking exposure, and thus may be useful for clinical decision making for lung adenocarcinoma and/or small cell lung carcinoma.

\section{Supplementary information}

Supplementary information accompanies this paper at https://doi.org/10. 1186/s12885-020-07407-x.

Additional file 1: Table S1. Characteristics of lung cancer cases ( $n=$ 372) by their quintiles of cg05575921 percent methylation. Table S2. Linear regression results for quintile cg05575921 hypomethylation and smoking features. Table S3. Lung cancer risk by cg05575921 percent methylation for all lung cancer cases and by histotype, excluding $n=3$ case/control pairs where one had 5PRIME DNA extraction. Table S4. 
Lung cancer risk by cg05575921 percent methylation, restricted to 2014 USPSTF lung cancer screening-eligible pairs, excluding $n=1$ case/control pair where one had 5PRIME DNA extraction. Table S5. Mortality by cg05575921 percent methylation for all lung cancer cases and by histotype, excluding $n=2$ participants with 5PRIME DNA extraction.

Additional file 2. Participating CARET Institutions and Federalwide Assurance Numbers by Study Center.

\section{Abbreviations}

AHRR: Aryl-hydrocarbon receptor repressor gene; BMI: Body mass index; CARET: $\beta$-Carotene and Retinol Efficacy Trial; Cl: Confidence interval; CpG: Cytosine-guanine dinucleotide; EWAS: Epigenome-wide association studies; HR: Hazard ratio; NSCLC: Non-small cell lung cancer; NSCLC, NOS: Non-small cell lung cancer, not otherwise specified; PAH: Polycyclic aromatic hydrocarbon; OR: Odds ratio; SD: Standard deviation; USPSTF: United States Preventive Services Task Force

\section{Acknowledgements}

Not applicable.

\section{Authors' contributions}

$L G, S G, D C K, C J M$, and JAD designed the investigation. CC, GEG, MDT, and MJB designed the CARET study and oversee use of CARET data. CJM and JAD generated DNA methylation data for CARET samples. DCK processed, performed quality control, and generated preliminary analyses of DNA methylation data. $\mathrm{LG}, \mathrm{SG}$, and DCK analyzed the data. $\mathrm{LG}, \mathrm{SG}, \mathrm{DCK}, \mathrm{CJM}, \mathrm{DCK}$, and JAD interpreted the results. $L G$ and JAD drafted the manuscript, and $L G$, SG, MJB, CC, DCK CJM, and JAD edited the manuscript. All authors read and approved the final manuscript.

\section{Authors' information}

Not applicable

\section{Funding}

The research reported in this publication was supported by the National Center for Advancing Translational Sciences (NCATS) of the NIH under Award Number TL1 TR002540 and the National Cancer Institute (NCl) of the NIH R01 CA151989 (to J.A. Doherty), the Munck-Pfefferkorn Fund at Dartmouth College (to J.A. Doherty and C.J. Marsit), the Huntsman Cancer Foundation (to J.A. Doherty), and the Kansas IDeA Network of Biomedical Research Excellence Bioinformatics Core (to D.C. Koestler), and supported in part by the National Institute of General Medical Science (NIGMS) award P20 GM103418 (to D.E. Wright), and the NCl under award numbers P30 CA042014 (to M.E. Beckerle), P30 CA168524 (to R.A. Jensen), and R01 CA111703 (to C. Chen). Support for CARET is from NCl grants UM1 CA167462 and U01 CA63673 (to G.E. Goodman) and U01 CA167462 (to C. Chen). The funding bodies had no roles in the design of the study and collection, analysis, and interpretation of data and in writing the manuscript. The content is solely the responsibility of the authors and does not necessarily represent the official views of the National Institutes of Health.

\section{Availability of data and materials}

The data that support the findings of this study are available from CARET but restrictions apply to the availability of these data, which were used in agreement with CARET for the current study, and so are not publicly available. Data are available from the authors upon request and with permission of CARET (http://www.compass.fhcrc.org/caretWeb/requests/ requesting.aspx).

\section{Ethics approval and consent to participate}

All procedures performed in studies involving human participants were in accordance with the ethical standards of the Institutional Review Boards for each participating CARET institution (full list by study site, including Federalwide Assurance Numbers, are included in Additional file 2), overseen by the CARET Coordinating Center (Fred Hutchinson Cancer Research Center, Seattle, WA), and with the 1964 Helsinki declaration and its later amendments or comparable ethical standards. Written informed consent was obtained from all CARET participants.

\section{Consent for publication}

Not applicable.

\section{Competing interests}

The authors declare that they have no competing interests.

\section{Author details}

${ }^{1}$ Department of Population Health Sciences, Huntsman Cancer Institute, University of Utah, 2000 Circle of Hope Drive, Room 4746, Salt Lake City, UT 84112, USA. ²Department of Biostatistics \& Data Science, University of Kansas Medical Center, Kansas City, KS, USA. ${ }^{3}$ Department of Environmental Health, Rollins School of Public Health, Emory University, Atlanta, GA, USA. ${ }^{4}$ Program in Epidemiology, Division of Public Health Sciences, Fred Hutchinson Cancer Research Center, Seattle, WA, USA. ${ }^{5}$ Department of Epidemiology, School of Public Health, University of Washington, Seattle, WA, USA. ${ }^{6}$ Department of Otolaryngology: Head and Neck Surgery, School of Medicine, University of Washington, Seattle, WA, USA.

Received: 1 July 2020 Accepted: 14 September 2020

Published online: 22 September 2020

\section{References}

1. EWAS. Atlas: a curated knowledgebase of epigenome-wide association studies. Nucleic Acids Res. 2019;47(D1):D983-D8.

2. Gao X, Jia M, Zhang Y, Breitling LP, Brenner H. DNA methylation changes of whole blood cells in response to active smoking exposure in adults: a systematic review of DNA methylation studies. Clin Epigenetics. 2015;7:113.

3. Joehanes R, Just AC, Marioni RE, Pilling LC, Reynolds LM, Mandaviya PR, et al. Epigenetic signatures of cigarette smoking. Circ Cardiovasc Genet. 2016;9(5):436-47.

4. Baglietto L, Ponzi E, Haycock P, Hodge A, Bianca Assumma M, Jung CH, et al. DNA methylation changes measured in pre-diagnostic peripheral blood samples are associated with smoking and lung cancer risk. Int J Cancer. 2017;140(1):50-61.

5. Wilson R, Wahl S, Pfeiffer L, Ward-Caviness CK, Kunze S, Kretschmer A, et al. The dynamics of smoking-related disturbed methylation: a two time-point study of methylation change in smokers, non-smokers and former smokers. BMC Genomics. 2017;18(1):805.

6. Philibert R, Dogan M, Noel A, Miller S, Krukow B, Papworth E, et al. Dose Response and Prediction Characteristics of a Methylation Sensitive Digital PCR Assay for Cigarette Consumption in Adults. Front Gen. 2018;9:137.

7. Bakulski KM, Dou J, Lin N, London SJ, Colacino JA. DNA methylation signature of smoking in lung cancer is enriched for exposure signatures in newborn and adult blood. Sci Rep. 2019;9(1):4576.

8. Li S, Wong EM, Bui M, Nguyen TL, Joo JE, Stone J, et al. Causal effect of smoking on DNA methylation in peripheral blood: a twin and family study. Clin Epigenetics. 2018;10:18.

9. Bojesen SE, Timpson N, Relton C, Davey Smith G, Nordestgaard BG. AHRR (cg05575921) hypomethylation marks smoking behaviour, morbidity and mortality. Thorax. 2017;72(7):646-53.

10. Philibert R, Hollenbeck N, Andersen E, Osborn T, Gerrard M, Gibbons FX, et al. A quantitative epigenetic approach for the assessment of cigarette consumption. Front Psychol. 2015;6:656.

11. McCartney DL, Stevenson AJ, Hillary RF, Walker RM, Bermingham ML, Morris SW, et al. Epigenetic signatures of starting and stopping smoking. EBioMedicine. 2018;37:214-20.

12. Zhang Y, Schöttker B, Florath I, Stock C, Butterbach $K$, Holleczek B, et al. Smoking-associated DNA methylation biomarkers and their predictive value for all-cause and cardiovascular mortality. Environ Health Perspect. 2016; 124(1):67-74.

13. Monick MM, Beach SR, Plume J, Sears R, Gerrard M, Brody GH, et al. Coordinated changes in AHRR methylation in lymphoblasts and pulmonary macrophages from smokers. Am J Med Genet B Neuropsychiatr Genet. 2012;159B(2):141-51.

14. Reynolds LM, Wan M, Ding J, Taylor JR, Lohman K, Su D, et al. DNA methylation of the aryl hydrocarbon receptor repressor associations with cigarette smoking and subclinical atherosclerosis. Circ Cardiovasc Genet. 2015;8(5):707-16.

15. de Vries M, van der Plaat DA, Nedeljkovic I, Verkaik-Schakel RN, Kooistra W, Amin $\mathrm{N}$, et al. From blood to lung tissue: effect of cigarette smoke on DNA methylation and lung function. Respir Res. 2018;19(1):212. 
16. Shenker NS, Polidoro S, van Veldhoven K, Sacerdote C, Ricceri F, Birrell MA et al. Epigenome-wide association study in the European prospective investigation into Cancer and nutrition (EPIC-Turin) identifies novel genetic loci associated with smoking. Hum Mol Genet. 2013;22(5):843-51.

17. Stueve TR, Li W-Q, Shi J, Marconett CN, Zhang T, Yang C, et al. Epigenomewide analysis of DNA methylation in lung tissue shows concordance with blood studies and identifies tobacco smoke-inducible enhancers. Hum Mol Genet. 2017;26(15):3014-27.

18. Tsay JJ, Tchou-Wong K-M, Greenberg AK, Pass H, Rom WN. Aryl hydrocarbon receptor and lung cancer. Anticancer Res. 2013;33(4): 1247-56.

19. Fasanelli F, Baglietto L, Ponzi E, Guida F, Campanella G, Johansson M, et al. Hypomethylation of smoking-related genes is associated with future lung cancer in four prospective cohorts. Nat Commun. 2015;6:10192.

20. Zhang Y, Elgizouli M, Schöttker B, Holleczek B, Nieters A, Brenner H. Smoking-associated DNA methylation markers predict lung cancer incidence. Clin Epigenetics. 2016;8(1):127.

21. Battram T, Richmond RC, Baglietto L, Haycock PC, Perduca V, Bojesen SE, et al. Appraising the causal relevance of DNA methylation for risk of lung cancer. Int J Epidemiol. 2019;48(5):1493-504.

22. Sandanger TM, Nøst TH, Guida F, Rylander C, Campanella G, Muller DC, et al. DNA methylation and associated gene expression in blood prior to lung cancer diagnosis in the Norwegian women and Cancer cohort. Sci Rep. 2018:8(1):16714

23. Zhang Y, Breitling LP, Balavarca Y, Holleczek B, Schottker B, Brenner H. Comparison and combination of blood DNA methylation at smokingassociated genes and at lung cancer-related genes in prediction of lung cancer mortality. Int J Cancer. 2016;139(11):2482-92.

24. Moyer VA. Screening for lung cancer: U.S. preventive services task force recommendation statement. Ann Intern Med. 2014;160(5):330-8.

25. Draft Recommendation Statement: Lung Cancer: Screening. U.S. Preventive Services Task Force July 07, 2020.

26. Omenn GS, Goodman GE, Thornquist MD, Balmes J, Cullen MR, Glass A, et al. Risk factors for lung cancer and for intervention effects in CARET, the Betacarotene and retinol efficacy trial. J Natl Cancer Inst. 1996;88(21):1550-9.

27. Goodman GE, Thornquist MD, Balmes J, Cullen MR, Meyskens FL Jr, Omenn GS, et al. The Beta-carotene and retinol efficacy trial: incidence of lung cancer and cardiovascular disease mortality during 6-year follow-up after stopping betacarotene and retinol supplements. J Natl Cancer Inst. 2004;96(23):1743-50.

28. Omenn GS, Goodman GE, Thornquist MD, Balmes J, Cullen MR, Glass A et al. Effects of a combination of beta carotene and vitamin a on lung cancer and cardiovascular disease. N Engl J Med. 1996;334(18):1150-5.

29. Sakoda L, Loomis M, Doherty J, Julianto L, Barnett M, Neuhouser M, et al. Germ line variation in nucleotide excision repair genes and lung cancer risk in smokers. Int J Mol Epidemiol Genet. 2012;3(1):1-17.

30. Aryee MJ, Jaffe AE, Corrada-Bravo H, Ladd-Acosta C, Feinberg AP, Hansen $K D$, et al. Minfi: a flexible and comprehensive bioconductor package for the analysis of Infinium DNA methylation microarrays. Bioinformatics. 2014; 30(10):1363-9.

31. Pidsley R, CC YW, Volta M, Lunnon K, Mill J, Schalkwyk LC. A data-driven approach to preprocessing Illumina 450K methylation array data. BMC Genomics. 2013;14:293.

32. Grieshober L, Graw S, Barnett MJ, Thornquist MD, Goodman GE, Chen C, et al. Methylation-derived neutrophil-to-lymphocyte ratio and lung Cancer risk in heavy smokers. Cancer Prev Res. 2018;11(11):727-34.

33. Koestler DC, Jones MJ, Usset J, Christensen BC, Butler RA, Kobor MS, et al. Improving cell mixture deconvolution by identifying optimal DNA methylation libraries (IDOL). BMC Bioinformatics. 2016;17:120.

34. Houseman EA, Accomando WP, Koestler DC, Christensen BC, Marsit CJ, Nelson $\mathrm{HH}$, et al. DNA methylation arrays as surrogate measures of cell mixture distribution. BMC Bioinformatics. 2012;13:86.

35. Paesmans M. Prognostic and predictive factors for lung cancer. Breathe. 2012;9(2):112-21.

36. Zeilinger S, Kühnel B, Klopp N, Baurecht H, Kleinschmidt A, Gieger C, et al. Tobacco smoking leads to extensive genome-wide changes in DNA methylation. PLoS One. 2013;8(5):e63812.

37. Guida F, Sandanger TM, Castagné R, Campanella G, Polidoro S, Palli D, et al. Dynamics of smoking-induced genome-wide methylation changes with time since smoking cessation. Hum Mol Genet. 2015;24(8):2349-59.
38. Groen K, Lea RA, Maltby VE, Scott RJ, Lechner-Scott J. Letter to the editor: blood processing and sample storage have negligible effects on methylation. Clin Epigenetics. 2018;10(1):22.

39. Li Y, Pan X, Roberts ML, Liu P, Kotchen TA, Cowley AW Jr, et al. Stability of global methylation profiles of whole blood and extracted DNA under different storage durations and conditions. Epigenomics. 2018;10(6):797-811.

40. Hjorthaug HS, Gervin K, Mowinckel P, Munthe-Kaas MC. Exploring the influence from whole blood DNA extraction methods on Infinium 450K DNA methylation. PLoS One. 2018;13(12):e0208699.

41. Bibikova M, Barnes B, Tsan C, Ho V, Klotzle B, Le JM, et al. High density DNA methylation array with single CpG site resolution. Genomics. 2011;98(4):288-95.

\section{Publisher's Note}

Springer Nature remains neutral with regard to jurisdictional claims in published maps and institutional affiliations.

\section{Ready to submit your research? Choose BMC and benefit from:}

- fast, convenient online submission

- thorough peer review by experienced researchers in your field

- rapid publication on acceptance

- support for research data, including large and complex data types

- gold Open Access which fosters wider collaboration and increased citations

- maximum visibility for your research: over $100 \mathrm{M}$ website views per year

At $\mathrm{BMC}$, research is always in progress.

Learn more biomedcentral.com/submissions 